Supporting Information

\title{
Dissolution Kinetics of a BCS Class II Active Pharmaceutical Ingredient: Diffusion-Based Model Validation and Prediction
}

Yuan Gao a, Brian Glennon a,b, Yunliang He a, Philip Donnellan a*

a Synthesis and Solid State Pharmaceutical Centre (SSPC), School of Chemical and Bioprocess Engineering, University College Dublin, Belfield, Dublin 4, Ireland

b APC Ltd, Building 11 Cherrywood Business Park, Loughlinstown, Dublin 18, Ireland 


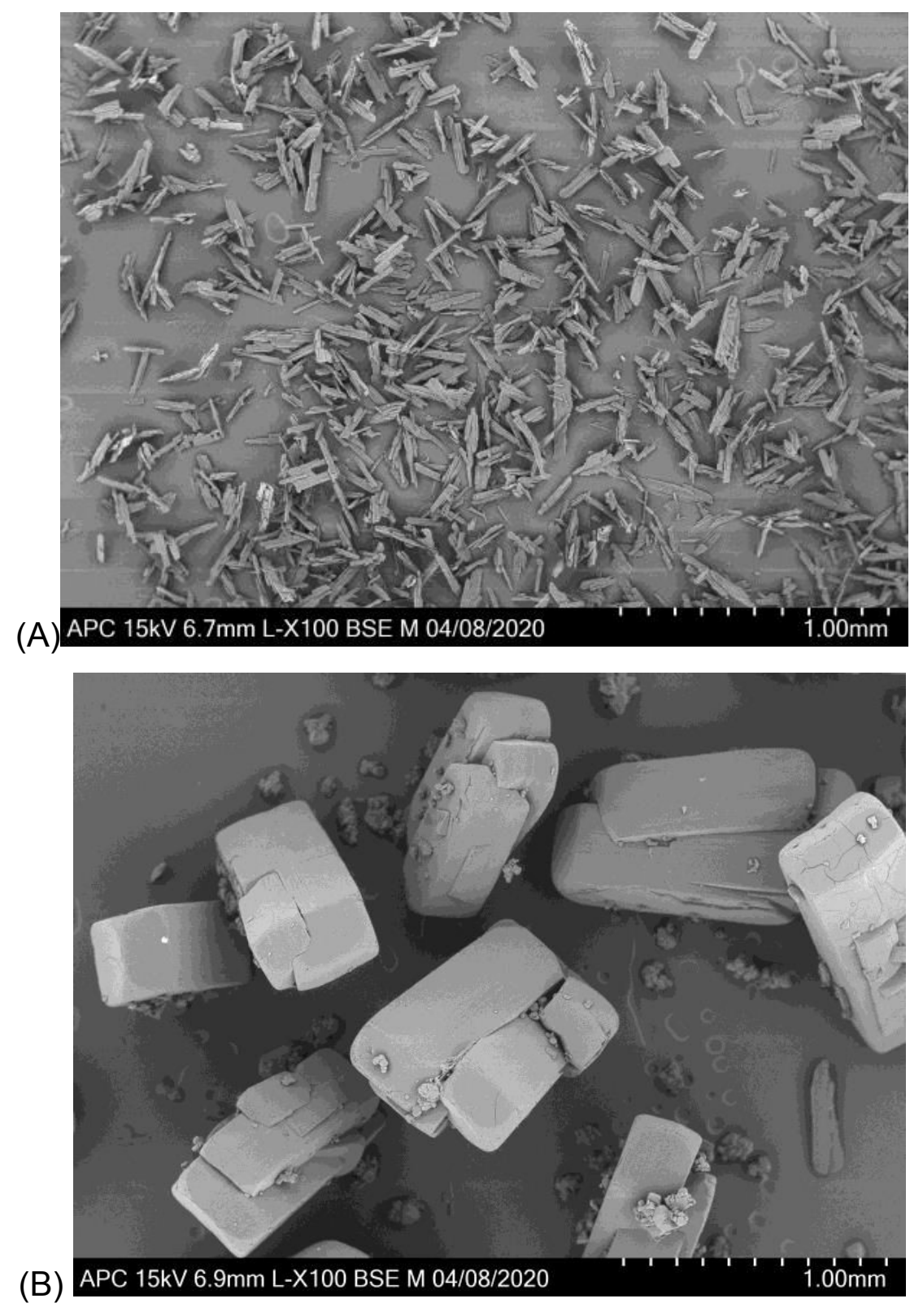

Figure S1. SEM images of ibuprofen crystals for dissolution: (A) needle-like; (B) polyhedral. 


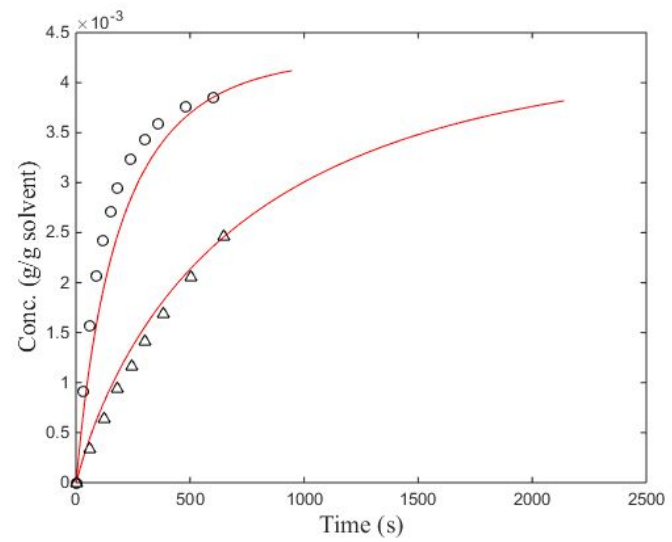

(A)

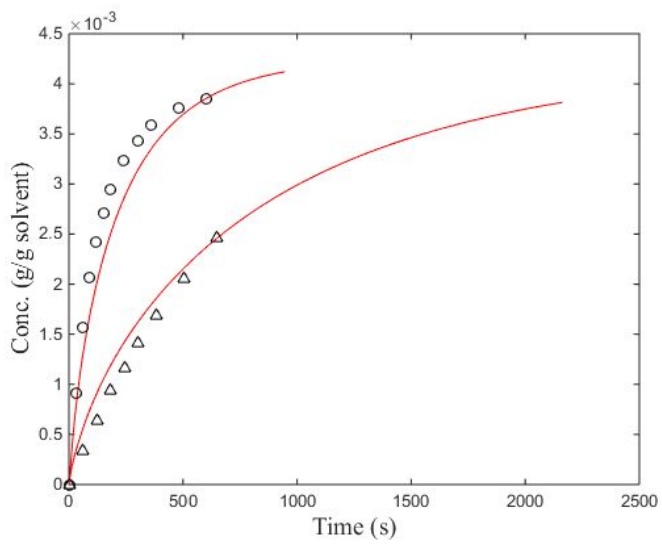

(C)

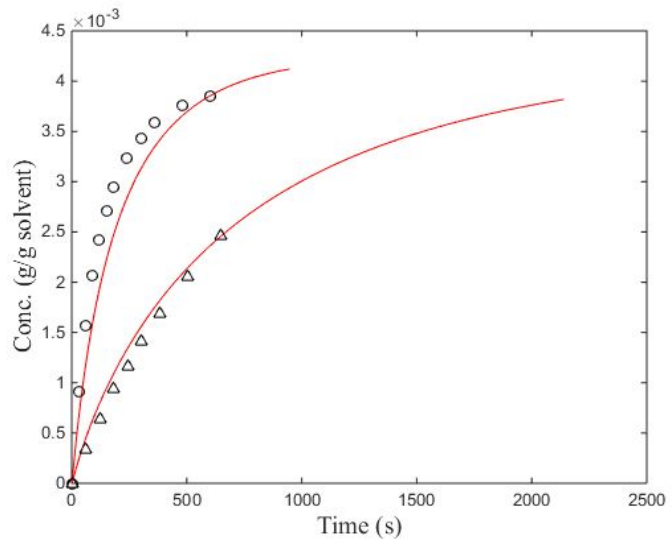

(B)

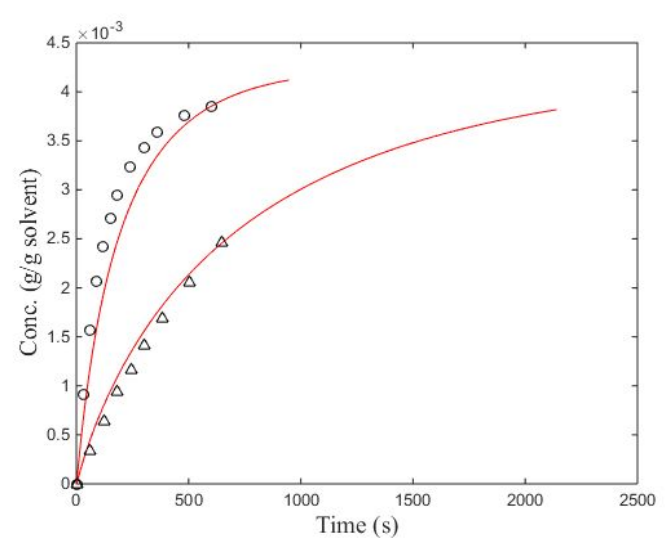

(D)

Figure S2. Experimental dissolution profiles of fine (o) and coarse $(\Delta)$ ibuprofen when $R=100 \mathrm{rpm}$. Simulated curves were drawn using spherical geometry with (A) size-independent $h \&$ (B) size-dependent $h$, and cylindrical geometry with (C) size-independent $h \&(\mathrm{D})$ size-dependent $h$. 


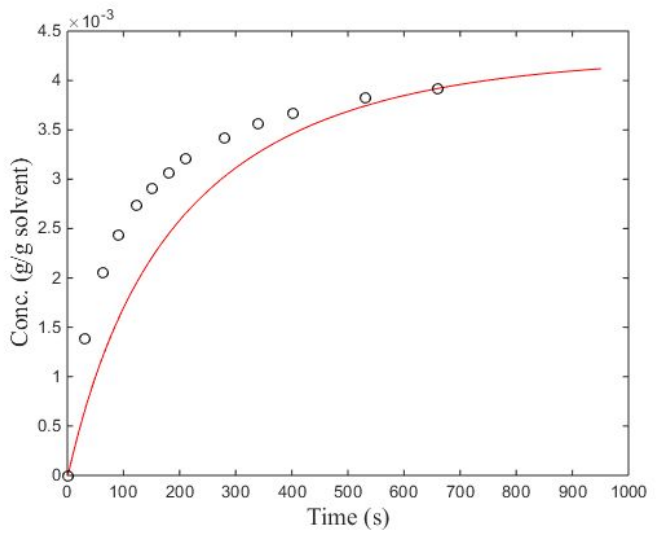

(A)

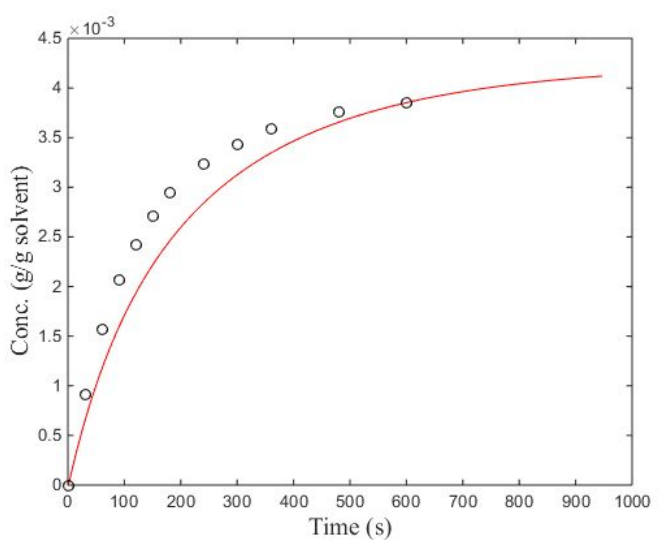

(C)

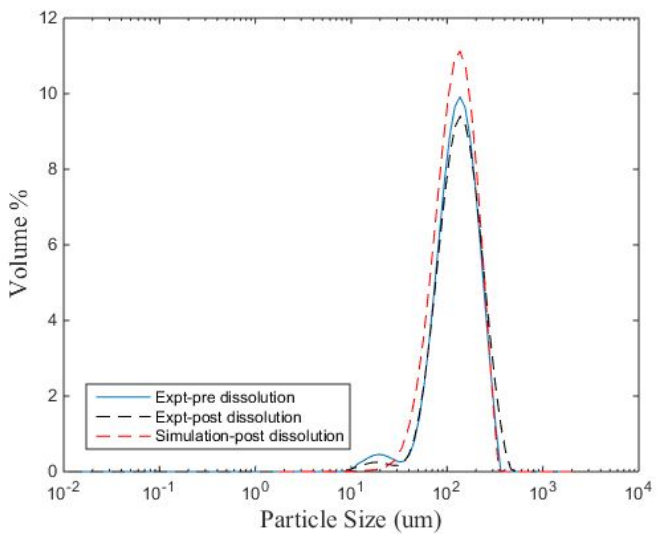

(B)

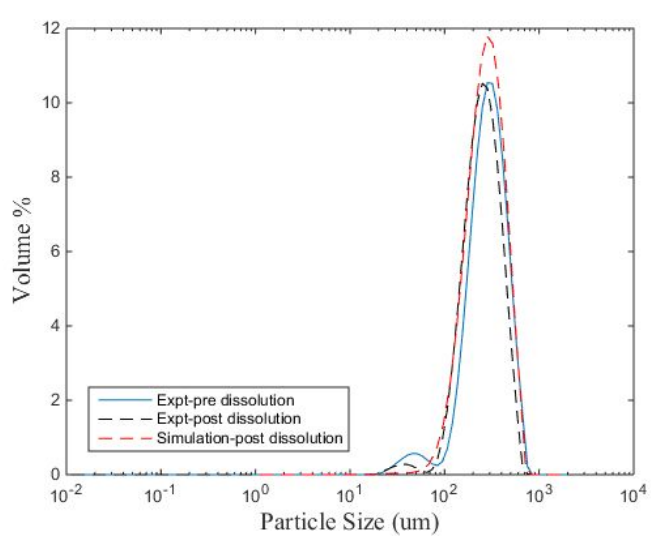

(D)

Figure S3. Cylindrical geometry model validation with Run 1 data (dissolution profiles and CSDs). Simulated red curves were drawn using size-independent $h$ in $(\mathrm{A}) \&(\mathrm{~B})$ and size-dependent $h$ in (C)\&(D). 


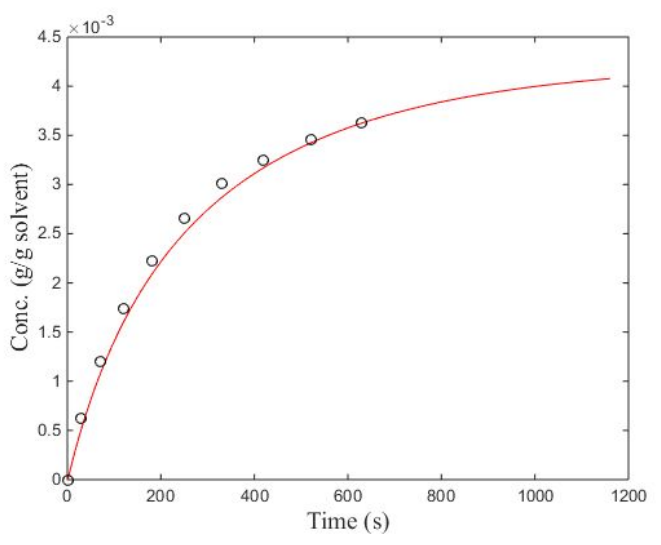

(A)

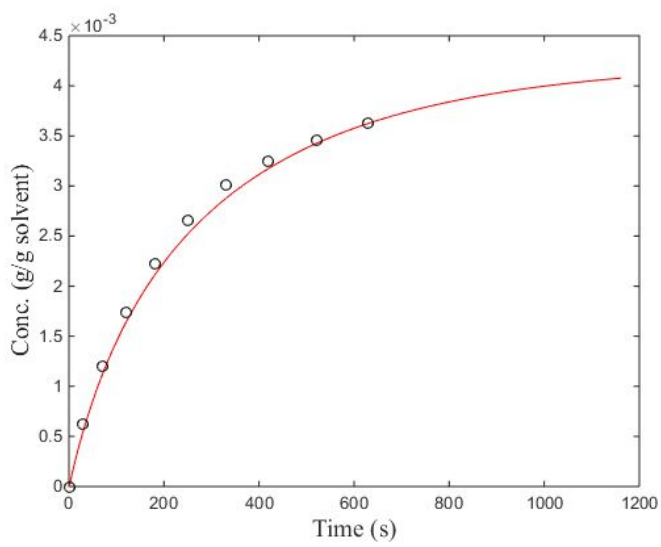

(C)

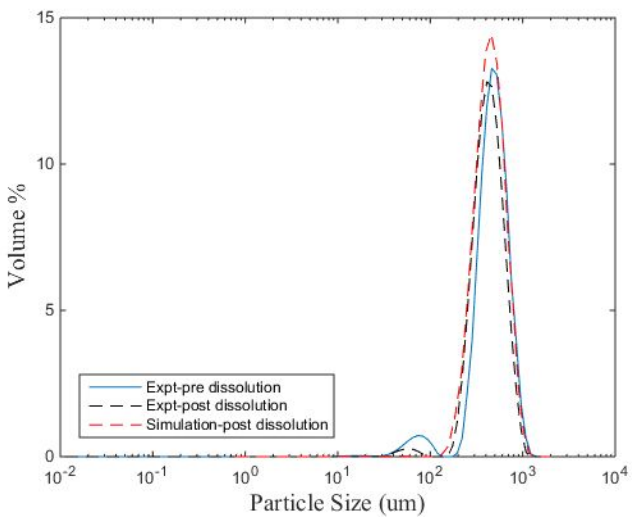

(B)

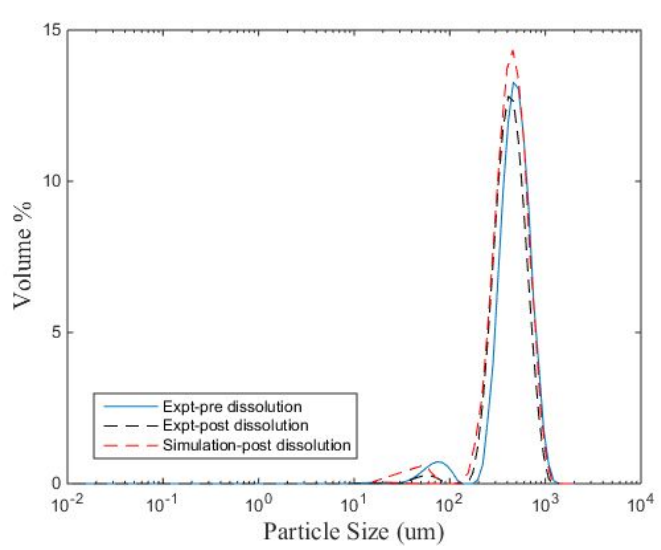

(D)

Figure S4. Cylindrical geometry model validation with Run 3 data (dissolution profiles and CSDs). Simulated red curves were drawn using size-independent $h$ in (A)\&(B) and size-dependent $h$ in (C)\&(D). 


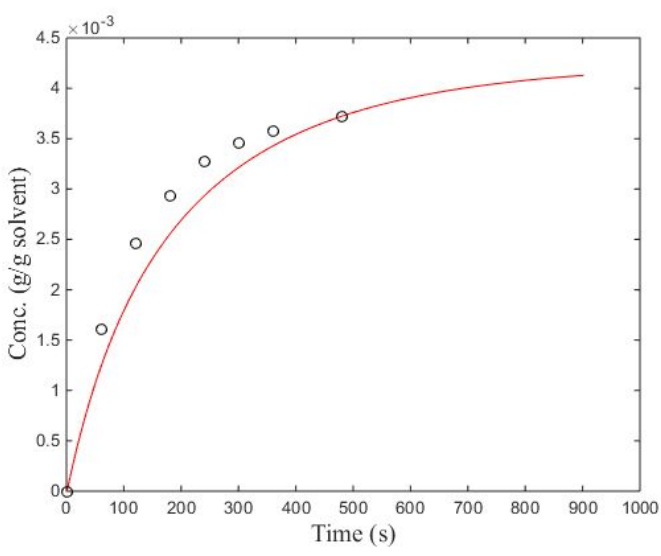

(A)

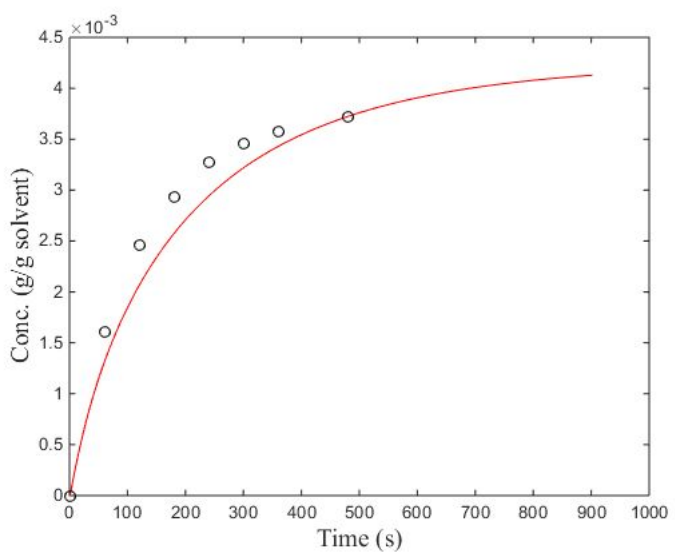

(C)

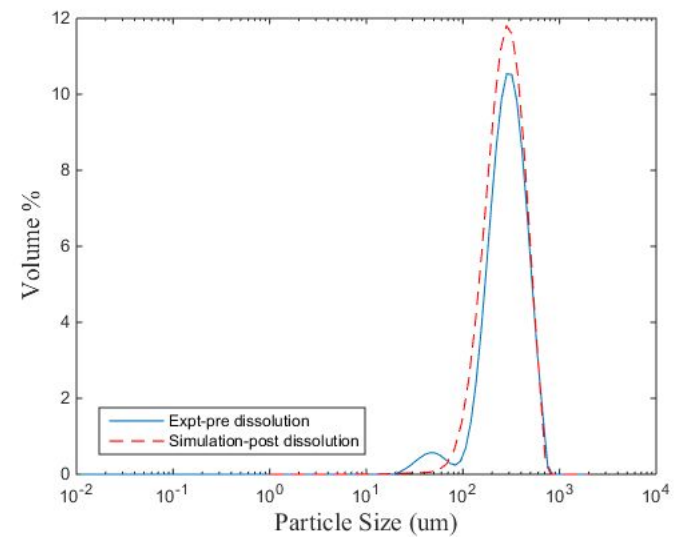

(B)

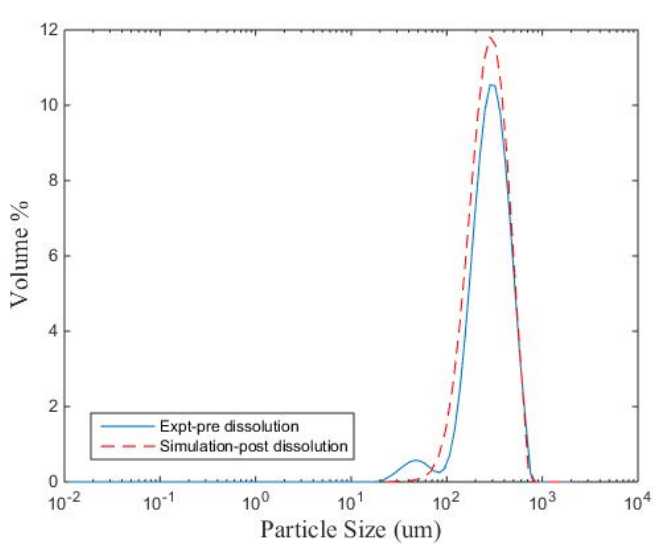

(D)

Figure S5. Cylindrical geometry model validation with Run 5 data (dissolution profiles and CSDs). Simulated red curves were drawn using size-independent $h$ in $(\mathrm{A}) \&(\mathrm{~B})$ and size-dependent $h$ in (C)\&(D). 


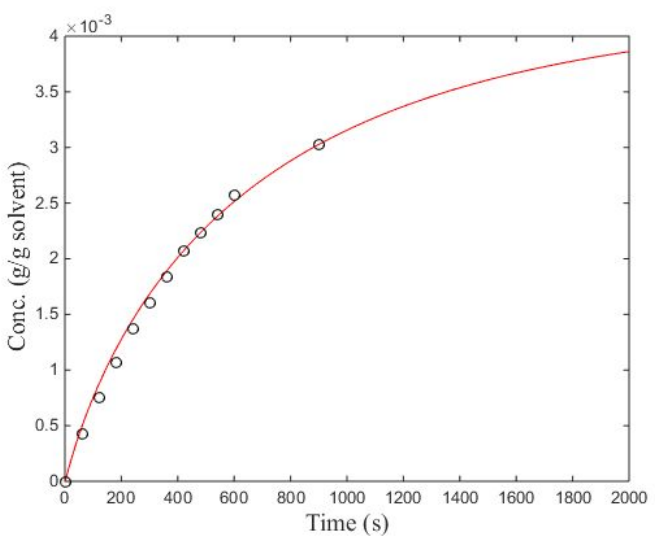

(A)

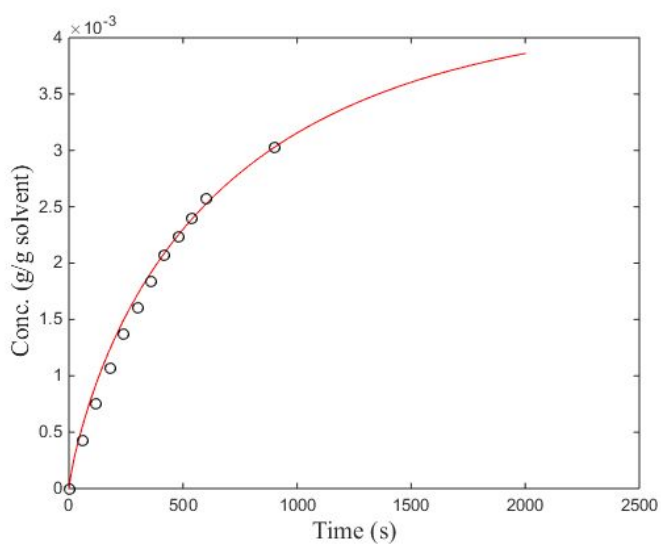

(C)

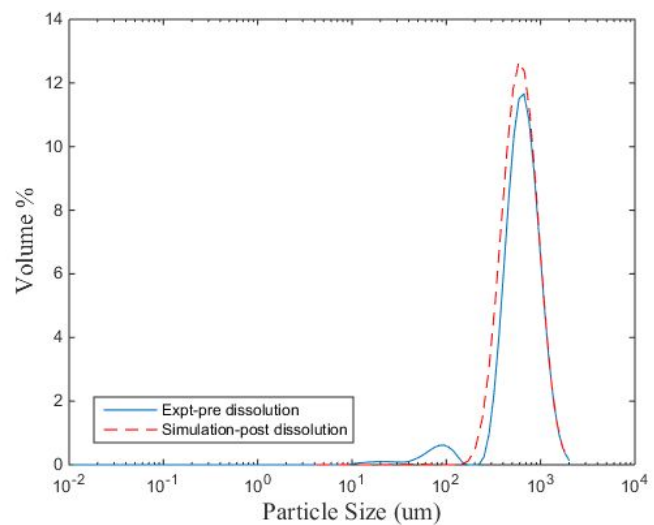

(B)

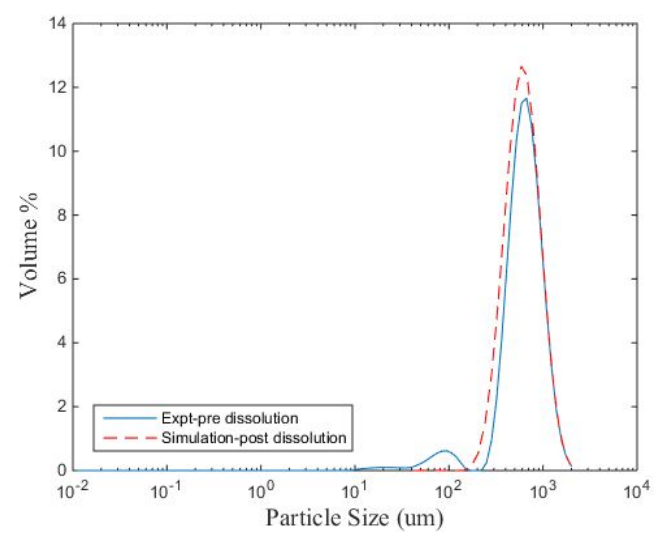

(D)

Figure S6. Cylindrical geometry model validation with Run 7 data (dissolution profiles and CSDs). Simulated red curves were drawn using size-independent $h$ in (A)\&(B) and size-dependent $h$ in (C)\&(D). 


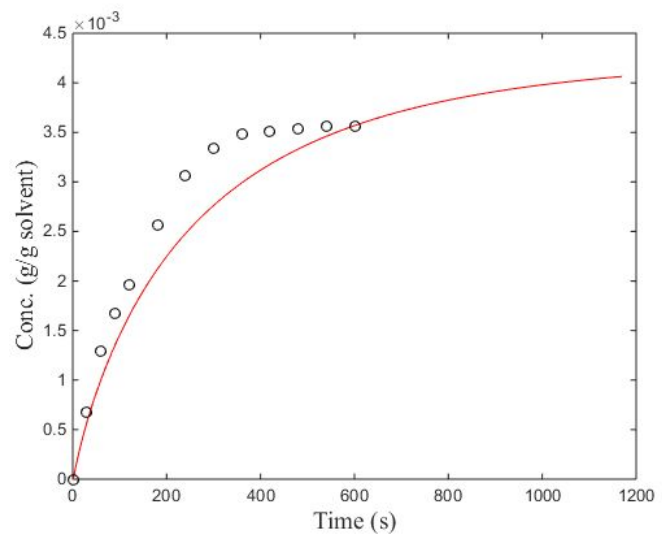

(A)

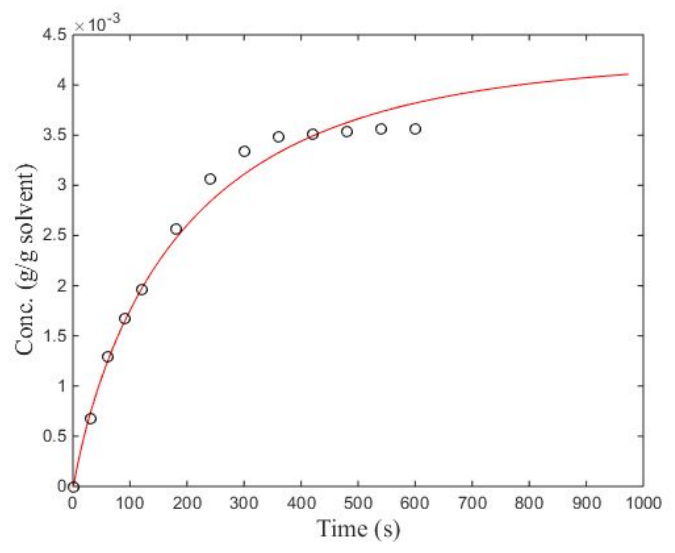

(C)

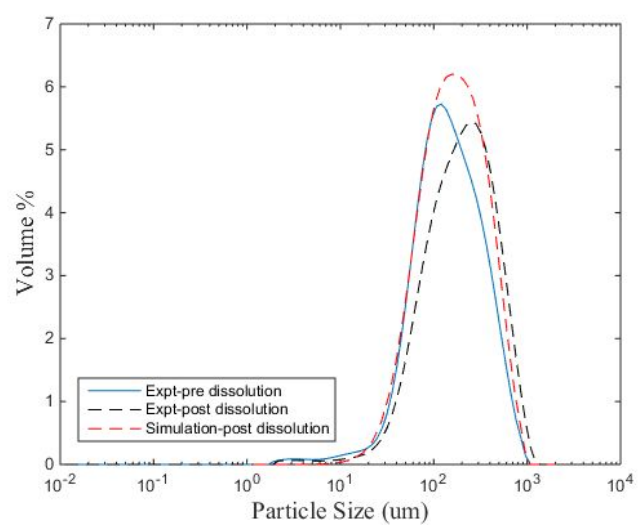

(B)

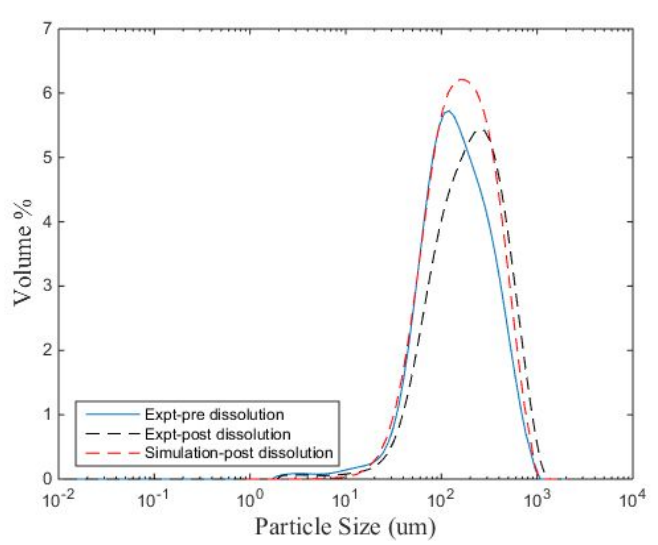

(D)

Figure S7. Cylindrical geometry model validation with Run 10 data (dissolution profiles and CSDs). Simulated red curves were drawn using size-independent $h$ in $(\mathrm{A}) \&(\mathrm{~B})$ and size-dependent $h$ in (C)\&(D). 
Table S1. Experimental dissolution profiles of ibuprofen in phosphate buffer $(\mathrm{pH}=7.20)$ at $\mathrm{T}=37^{\circ} \mathrm{C}$.

\begin{tabular}{|c|c|c|c|}
\hline$t / s$ & $y^{\exp }$ & $t / s$ & $y^{\exp }$ \\
\hline \multicolumn{2}{|c|}{$\begin{array}{l}\text { Run1: } L<150 \mu \mathrm{m}, R=100 \mathrm{rpm}, \\
\text { Morphology=Polyhedral, }-\sigma=1\end{array}$} & \multicolumn{2}{|c|}{$\begin{array}{l}\text { Run2: } L=150-300 \mu \mathrm{m}, R=100 \mathrm{rpm} \text {, } \\
\text { Morphology=Polyhedral, }-\sigma=1\end{array}$} \\
\hline 0 & 0 & 0 & 0 \\
\hline 30.46 & 0.3271 & 30.24 & 0.2136 \\
\hline 63.42 & 0.4822 & 60.32 & 0.3702 \\
\hline 90.23 & 0.573 & 90.22 & 0.4863 \\
\hline 123.28 & 0.6436 & 120.22 & 0.5702 \\
\hline 150.79 & 0.6839 & 150.3 & 0.6383 \\
\hline 180.22 & 0.7203 & 180.16 & 0.6916 \\
\hline 210.35 & 0.7534 & 240.26 & 0.7621 \\
\hline 280.66 & 0.8042 & 301.15 & 0.8079 \\
\hline 340.99 & 0.8383 & 360.39 & 0.843 \\
\hline 401.15 & 0.862 & 480.32 & 0.8838 \\
\hline 530.67 & 0.9004 & 600.61 & 0.9064 \\
\hline 660.21 & 0.9221 & & \\
\hline \multicolumn{2}{|c|}{$\begin{array}{c}\text { Run3: } L=300-500 \mu \mathrm{m}, R=100 \mathrm{rpm}, \\
\text { Morphology=Polyhedral, }-\sigma=1\end{array}$} & \multicolumn{2}{|c|}{$\begin{array}{c}\text { Run4: } L=500-850 \mu \mathrm{m}, R=100 \mathrm{rpm} \text {, } \\
\text { Morphology=Polyhedral, }-\sigma=1\end{array}$} \\
\hline 0 & 0 & 0 & 0 \\
\hline 30.29 & 0.147 & 60.32 & 0.078 \\
\hline 70.27 & 0.2839 & 120.68 & 0.1482 \\
\hline 120.45 & 0.4083 & 182.43 & 0.2194 \\
\hline 180.59 & 0.5241 & 242.77 & 0.2728 \\
\hline
\end{tabular}




\begin{tabular}{|c|c|c|c|}
\hline 250.83 & 0.6241 & 303.1 & 0.3323 \\
\hline 330.38 & 0.7077 & 383.42 & 0.3956 \\
\hline 420.29 & 0.7652 & 504.15 & 0.4832 \\
\hline 520.23 & 0.8146 & 648.91 & 0.578 \\
\hline 630.49 & 0.8529 & & \\
\hline \multicolumn{2}{|c|}{$\begin{array}{l}\text { Run5: } L=150-300 \mu \mathrm{m}, R=150 \mathrm{rpm} \\
\text { Morphology=Polyhedral, }-\sigma=1\end{array}$} & \multicolumn{2}{|c|}{$\begin{array}{c}\text { Run6: } L=300-500 \mu \mathrm{m}, R=150 \mathrm{rpm} \\
\text { Morphology=Polyhedral, }-\sigma=1\end{array}$} \\
\hline 0 & 0 & 0 & 0 \\
\hline 60 & 0.379 & 30 & 0.1321 \\
\hline 120 & 0.5799 & 60 & 0.2447 \\
\hline 180 & 0.6906 & 90 & 0.3343 \\
\hline 240 & 0.7688 & 120 & 0.4122 \\
\hline 300 & 0.8119 & 180 & 0.5298 \\
\hline 360 & 0.84 & 240 & 0.6246 \\
\hline 420 & 0.8481 & 300 & 0.6869 \\
\hline 480 & 0.8759 & 360 & 0.7348 \\
\hline 540 & 0.8744 & 420 & 0.7729 \\
\hline \multirow[t]{3}{*}{600} & 0.8752 & 480 & 0.7931 \\
\hline & & 540 & 0.8114 \\
\hline & & 600 & 0.8272 \\
\hline \multicolumn{2}{|c|}{$\begin{array}{l}\text { Run7: } L=500-850 \mu \mathrm{m}, R=150 \mathrm{rpm}, \\
\text { Morphology=Polyhedral, }-\sigma=1\end{array}$} & \multicolumn{2}{|c|}{$\begin{array}{l}\text { Run8: } L>850 \mu \mathrm{m}, R=150 \mathrm{rpm} \text {, } \\
\text { Morphology=Polyhedral, }-\sigma=1\end{array}$} \\
\hline 0.00 & 0.0000 & 0.00 & 0.0000 \\
\hline 60.00 & 0.1011 & 60.00 & 0.0630 \\
\hline
\end{tabular}




$\begin{array}{ll}120.00 & 0.1770 \\ 180.00 & 0.2524 \\ 240.00 & 0.3213 \\ 300.00 & 0.3777 \\ 360.00 & 0.4311 \\ 420.00 & 0.4877 \\ 480.00 & 0.5241 \\ 540.00 & 0.5641 \\ 600.00 & 0.6040\end{array}$

\begin{tabular}{|ll}
120.00 & 0.1368 \\
180.00 & 0.1971 \\
240.00 & 0.2548 \\
300.00 & 0.3119 \\
360.00 & 0.3615 \\
420.00 & 0.4062 \\
480.00 & 0.4468 \\
540.00 & 0.4894 \\
600.00 & 0.5144
\end{tabular}

Run9: $L<150 \mu \mathrm{m}, R=100 \mathrm{rpm}$, Morphology $=$ Needle-like, $-\sigma=1$

$\begin{array}{cc}0 & 0 \\ 30.26 & 0.3739 \\ 60.31 & 0.5255 \\ 90.04 & 0.6241 \\ 120.22 & 0.7154 \\ 150.28 & 0.78 \\ 180.03 & 0.8369 \\ 240.19 & 0.8922 \\ 300.88 & 0.9314 \\ 360.29 & 0.9487 \\ 480.21 & 0.9749 \\ 601.17 & 0.9785\end{array}$

Run10: $L=150-300 \mu \mathrm{m}, R=100 \mathrm{rpm}$, Morphology $=$ Needle-like, $-\sigma=1$ 0

0

30

0.1594

60

0.3039

90

0.395

120

0.4607

180

0.6041

240

0.7216

300

0.7867

360

0.8211

420

0.8257

480

0.833

540

0.839

600

0.8396 


\begin{tabular}{cc|cc} 
Run11: $L=300-500 \mu \mathrm{m}, R=100 \mathrm{rpm}$, & \multicolumn{2}{c}{ Run12: $L=150-300 \mu \mathrm{m}, R=50 \mathrm{rpm}}$, \\
Habit=Needle-like, $-\sigma=1$ & \multicolumn{1}{c}{ Morphology=Polyhedral, $-\sigma=1$} \\
0 & 0 & 0 & 0 \\
30 & 0.0887 & 30.24 & 0.1158 \\
60 & 0.1472 & 65.75 & 0.1731 \\
90 & 0.2194 & 98.08 & 0.2398 \\
126 & 0.2981 & 125.21 & 0.2643 \\
180 & 0.4077 & 202.83 & 0.3312 \\
240 & 0.5154 & 299.85 & 0.384 \\
300 & 0.5905 & 360.39 & 0.4166 \\
360 & 0.6535 & 426.5 & 0.4498 \\
420 & 0.6801 & 547.33 & 0.5287 \\
480 & 0.7376 & 668.17 & 0.5843 \\
540 & 0.7686 & & \\
600 & 0.7959 & & \\
& & &
\end{tabular}


Table S2. List of ibuprofen CSDs before dissolution.

Particle

Volume

Size

Run Run Run Run Run Run Run Run Run Run Run

$\begin{array}{llllllllllll}(\mu \mathrm{m}) & 1 & 2 & 3 & 4 & 5 & 6 & 7 & 8 & 9 & 10 & 11\end{array}$

$\begin{array}{llllllllllll}0.01 & 0.00 & 0.00 & 0.00 & 0.00 & 0.00 & 0.00 & 0.00 & 0.00 & 0.00 & 0.00 & 0.00\end{array}$

$\begin{array}{llllllllllll}0.01 & 0.00 & 0.00 & 0.00 & 0.00 & 0.00 & 0.00 & 0.00 & 0.00 & 0.00 & 0.00 & 0.00\end{array}$

$\begin{array}{llllllllllll}0.01 & 0.00 & 0.00 & 0.00 & 0.00 & 0.00 & 0.00 & 0.00 & 0.00 & 0.00 & 0.00 & 0.00\end{array}$

$\begin{array}{llllllllllll}0.02 & 0.00 & 0.00 & 0.00 & 0.00 & 0.00 & 0.00 & 0.00 & 0.00 & 0.00 & 0.00 & 0.00\end{array}$

$\begin{array}{llllllllllll}0.02 & 0.00 & 0.00 & 0.00 & 0.00 & 0.00 & 0.00 & 0.00 & 0.00 & 0.00 & 0.00 & 0.00\end{array}$

$\begin{array}{llllllllllll}0.02 & 0.00 & 0.00 & 0.00 & 0.00 & 0.00 & 0.00 & 0.00 & 0.00 & 0.00 & 0.00 & 0.00\end{array}$

$\begin{array}{llllllllllll}0.02 & 0.00 & 0.00 & 0.00 & 0.00 & 0.00 & 0.00 & 0.00 & 0.00 & 0.00 & 0.00 & 0.00\end{array}$

$\begin{array}{llllllllllll}0.03 & 0.00 & 0.00 & 0.00 & 0.00 & 0.00 & 0.00 & 0.00 & 0.00 & 0.00 & 0.00 & 0.00\end{array}$

$\begin{array}{llllllllllll}0.03 & 0.00 & 0.00 & 0.00 & 0.00 & 0.00 & 0.00 & 0.00 & 0.00 & 0.00 & 0.00 & 0.00\end{array}$

$\begin{array}{llllllllllll}0.03 & 0.00 & 0.00 & 0.00 & 0.00 & 0.00 & 0.00 & 0.00 & 0.00 & 0.00 & 0.00 & 0.00\end{array}$

$\begin{array}{llllllllllll}0.04 & 0.00 & 0.00 & 0.00 & 0.00 & 0.00 & 0.00 & 0.00 & 0.00 & 0.00 & 0.00 & 0.00\end{array}$

$\begin{array}{llllllllllll}0.04 & 0.00 & 0.00 & 0.00 & 0.00 & 0.00 & 0.00 & 0.00 & 0.00 & 0.00 & 0.00 & 0.00\end{array}$

$\begin{array}{llllllllllll}0.05 & 0.00 & 0.00 & 0.00 & 0.00 & 0.00 & 0.00 & 0.00 & 0.00 & 0.00 & 0.00 & 0.00\end{array}$

$\begin{array}{llllllllllll}0.06 & 0.00 & 0.00 & 0.00 & 0.00 & 0.00 & 0.00 & 0.00 & 0.00 & 0.00 & 0.00 & 0.00\end{array}$

$\begin{array}{llllllllllll}0.06 & 0.00 & 0.00 & 0.00 & 0.00 & 0.00 & 0.00 & 0.00 & 0.00 & 0.00 & 0.00 & 0.00\end{array}$

$\begin{array}{llllllllllll}0.07 & 0.00 & 0.00 & 0.00 & 0.00 & 0.00 & 0.00 & 0.00 & 0.00 & 0.00 & 0.00 & 0.00\end{array}$

$\begin{array}{llllllllllll}0.08 & 0.00 & 0.00 & 0.00 & 0.00 & 0.00 & 0.00 & 0.00 & 0.00 & 0.00 & 0.00 & 0.00\end{array}$ 
$\begin{array}{llllllllllll}0.09 & 0.00 & 0.00 & 0.00 & 0.00 & 0.00 & 0.00 & 0.00 & 0.00 & 0.00 & 0.00 & 0.00\end{array}$

$\begin{array}{llllllllllll}0.10 & 0.00 & 0.00 & 0.00 & 0.00 & 0.00 & 0.00 & 0.00 & 0.00 & 0.00 & 0.00 & 0.00\end{array}$

$\begin{array}{llllllllllll}0.11 & 0.00 & 0.00 & 0.00 & 0.00 & 0.00 & 0.00 & 0.00 & 0.00 & 0.00 & 0.00 & 0.00\end{array}$

$\begin{array}{llllllllllll}0.13 & 0.00 & 0.00 & 0.00 & 0.00 & 0.00 & 0.00 & 0.00 & 0.00 & 0.00 & 0.00 & 0.00\end{array}$

$\begin{array}{llllllllllll}0.15 & 0.00 & 0.00 & 0.00 & 0.00 & 0.00 & 0.00 & 0.00 & 0.00 & 0.00 & 0.00 & 0.00\end{array}$

$\begin{array}{llllllllllll}0.17 & 0.00 & 0.00 & 0.00 & 0.00 & 0.00 & 0.00 & 0.00 & 0.00 & 0.00 & 0.00 & 0.00\end{array}$

$\begin{array}{llllllllllll}0.19 & 0.00 & 0.00 & 0.00 & 0.00 & 0.00 & 0.00 & 0.00 & 0.00 & 0.00 & 0.00 & 0.00\end{array}$

$\begin{array}{llllllllllll}0.21 & 0.00 & 0.00 & 0.00 & 0.00 & 0.00 & 0.00 & 0.00 & 0.00 & 0.00 & 0.00 & 0.00\end{array}$

$\begin{array}{llllllllllll}0.24 & 0.00 & 0.00 & 0.00 & 0.00 & 0.00 & 0.00 & 0.00 & 0.00 & 0.00 & 0.00 & 0.00\end{array}$

$\begin{array}{llllllllllll}0.27 & 0.00 & 0.00 & 0.00 & 0.00 & 0.00 & 0.00 & 0.00 & 0.00 & 0.00 & 0.00 & 0.00\end{array}$

$\begin{array}{llllllllllll}0.30 & 0.00 & 0.00 & 0.00 & 0.00 & 0.00 & 0.00 & 0.00 & 0.00 & 0.00 & 0.00 & 0.00\end{array}$

$\begin{array}{llllllllllll}0.34 & 0.00 & 0.00 & 0.00 & 0.00 & 0.00 & 0.00 & 0.00 & 0.00 & 0.00 & 0.00 & 0.00\end{array}$

$\begin{array}{llllllllllll}0.39 & 0.00 & 0.00 & 0.00 & 0.00 & 0.00 & 0.00 & 0.00 & 0.00 & 0.00 & 0.00 & 0.00\end{array}$

$\begin{array}{llllllllllll}0.44 & 0.00 & 0.00 & 0.00 & 0.00 & 0.00 & 0.00 & 0.00 & 0.00 & 0.00 & 0.00 & 0.00\end{array}$

$\begin{array}{llllllllllll}0.50 & 0.00 & 0.00 & 0.00 & 0.00 & 0.00 & 0.00 & 0.00 & 0.00 & 0.00 & 0.00 & 0.01\end{array}$

$\begin{array}{llllllllllll}0.56 & 0.00 & 0.00 & 0.00 & 0.00 & 0.00 & 0.00 & 0.00 & 0.00 & 0.00 & 0.00 & 0.07\end{array}$

$\begin{array}{llllllllllll}0.63 & 0.00 & 0.00 & 0.00 & 0.00 & 0.00 & 0.00 & 0.00 & 0.00 & 0.00 & 0.00 & 0.09\end{array}$

$\begin{array}{llllllllllll}0.72 & 0.00 & 0.00 & 0.00 & 0.00 & 0.00 & 0.00 & 0.00 & 0.00 & 0.00 & 0.00 & 0.11\end{array}$

$\begin{array}{llllllllllll}0.81 & 0.00 & 0.00 & 0.00 & 0.00 & 0.00 & 0.00 & 0.00 & 0.00 & 0.00 & 0.00 & 0.12\end{array}$

$\begin{array}{llllllllllll}0.91 & 0.00 & 0.00 & 0.00 & 0.00 & 0.00 & 0.00 & 0.00 & 0.00 & 0.00 & 0.00 & 0.13\end{array}$

$\begin{array}{llllllllllll}1.03 & 0.00 & 0.00 & 0.00 & 0.00 & 0.00 & 0.00 & 0.00 & 0.00 & 0.00 & 0.00 & 0.13\end{array}$

$\begin{array}{llllllllllll}1.17 & 0.00 & 0.00 & 0.00 & 0.00 & 0.00 & 0.00 & 0.00 & 0.00 & 0.00 & 0.00 & 0.14\end{array}$ 
$\begin{array}{llllllllllll}1.32 & 0.00 & 0.00 & 0.00 & 0.00 & 0.00 & 0.00 & 0.00 & 0.00 & 0.00 & 0.00 & 0.14\end{array}$

$\begin{array}{llllllllllll}1.49 & 0.00 & 0.00 & 0.00 & 0.00 & 0.00 & 0.00 & 0.00 & 0.00 & 0.02 & 0.00 & 0.15\end{array}$

$\begin{array}{llllllllllll}1.68 & 0.00 & 0.00 & 0.00 & 0.00 & 0.00 & 0.00 & 0.00 & 0.00 & 0.06 & 0.00 & 0.17\end{array}$

$\begin{array}{llllllllllll}1.90 & 0.00 & 0.00 & 0.00 & 0.00 & 0.00 & 0.00 & 0.00 & 0.00 & 0.08 & 0.04 & 0.18\end{array}$

$\begin{array}{llllllllllll}2.15 & 0.00 & 0.00 & 0.00 & 0.00 & 0.00 & 0.00 & 0.00 & 0.00 & 0.09 & 0.06 & 0.20\end{array}$

$\begin{array}{llllllllllll}2.43 & 0.00 & 0.00 & 0.00 & 0.00 & 0.00 & 0.00 & 0.00 & 0.00 & 0.11 & 0.08 & 0.22\end{array}$

$\begin{array}{llllllllllll}2.74 & 0.00 & 0.00 & 0.00 & 0.00 & 0.00 & 0.00 & 0.00 & 0.00 & 0.11 & 0.08 & 0.24\end{array}$

$\begin{array}{llllllllllll}3.10 & 0.00 & 0.00 & 0.00 & 0.00 & 0.00 & 0.00 & 0.00 & 0.00 & 0.12 & 0.09 & 0.26\end{array}$

$\begin{array}{llllllllllll}3.50 & 0.00 & 0.00 & 0.00 & 0.00 & 0.00 & 0.00 & 0.00 & 0.00 & 0.11 & 0.09 & 0.27\end{array}$

$\begin{array}{llllllllllll}3.96 & 0.00 & 0.00 & 0.00 & 0.00 & 0.00 & 0.00 & 0.00 & 0.00 & 0.11 & 0.08 & 0.29\end{array}$

$\begin{array}{llllllllllll}4.47 & 0.00 & 0.00 & 0.00 & 0.00 & 0.00 & 0.00 & 0.00 & 0.00 & 0.11 & 0.08 & 0.30\end{array}$

$\begin{array}{llllllllllll}5.05 & 0.00 & 0.00 & 0.00 & 0.00 & 0.00 & 0.00 & 0.00 & 0.00 & 0.11 & 0.08 & 0.31\end{array}$

$\begin{array}{llllllllllll}5.71 & 0.00 & 0.00 & 0.00 & 0.00 & 0.00 & 0.00 & 0.00 & 0.00 & 0.11 & 0.08 & 0.33\end{array}$

$\begin{array}{llllllllllll}6.45 & 0.00 & 0.00 & 0.00 & 0.00 & 0.00 & 0.00 & 0.00 & 0.00 & 0.12 & 0.08 & 0.35\end{array}$

$\begin{array}{llllllllllll}7.29 & 0.00 & 0.00 & 0.00 & 0.00 & 0.00 & 0.00 & 0.00 & 0.00 & 0.13 & 0.10 & 0.38\end{array}$

$\begin{array}{llllllllllll}8.23 & 0.00 & 0.00 & 0.00 & 0.00 & 0.00 & 0.00 & 0.00 & 0.00 & 0.15 & 0.11 & 0.42\end{array}$

$\begin{array}{llllllllllll}9.30 & 0.04 & 0.00 & 0.01 & 0.01 & 0.00 & 0.01 & 0.01 & 0.00 & 0.18 & 0.13 & 0.49\end{array}$

$\begin{array}{llllllllllll}10.51 & 0.10 & 0.00 & 0.02 & 0.04 & 0.00 & 0.02 & 0.04 & 0.00 & 0.20 & 0.15 & 0.59\end{array}$

$\begin{array}{llllllllllll}11.87 & 0.22 & 0.00 & 0.02 & 0.06 & 0.00 & 0.02 & 0.06 & 0.00 & 0.22 & 0.17 & 0.73\end{array}$

$\begin{array}{llllllllllll}13.42 & 0.29 & 0.00 & 0.03 & 0.07 & 0.00 & 0.03 & 0.07 & 0.00 & 0.25 & 0.18 & 0.91\end{array}$

$\begin{array}{llllllllllll}15.16 & 0.37 & 0.00 & 0.03 & 0.08 & 0.00 & 0.03 & 0.08 & 0.00 & 0.27 & 0.20 & 1.14\end{array}$

$\begin{array}{llllllllllll}17.12 & 0.43 & 0.00 & 0.03 & 0.09 & 0.00 & 0.03 & 0.09 & 0.00 & 0.31 & 0.22 & 1.41\end{array}$ 
$\begin{array}{llllllllllll}19.35 & 0.46 & 0.01 & 0.03 & 0.10 & 0.01 & 0.03 & 0.10 & 0.00 & 0.36 & 0.26 & 1.72\end{array}$ $\begin{array}{llllllllllll}21.86 & 0.44 & 0.06 & 0.03 & 0.10 & 0.06 & 0.03 & 0.10 & 0.00 & 0.47 & 0.32 & 2.05\end{array}$ $\begin{array}{llllllllllll}24.70 & 0.39 & 0.13 & 0.02 & 0.09 & 0.13 & 0.02 & 0.09 & 0.00 & 0.63 & 0.41 & 2.38\end{array}$ $\begin{array}{llllllllllll}27.90 & 0.31 & 0.21 & 0.02 & 0.09 & 0.21 & 0.02 & 0.09 & 0.00 & 0.89 & 0.57 & 2.68\end{array}$ $\begin{array}{llllllllllll}31.53 & 0.26 & 0.33 & 0.04 & 0.08 & 0.33 & 0.04 & 0.08 & 0.00 & 1.26 & 0.80 & 2.92\end{array}$ $\begin{array}{llllllllllll}35.62 & 0.28 & 0.43 & 0.08 & 0.08 & 0.43 & 0.08 & 0.08 & 0.00 & 1.75 & 1.13 & 3.09\end{array}$ $\begin{array}{llllllllllll}40.24 & 0.44 & 0.52 & 0.16 & 0.11 & 0.52 & 0.16 & 0.11 & 0.00 & 2.39 & 1.55 & 3.15\end{array}$ $\begin{array}{llllllllllll}45.47 & 0.80 & 0.57 & 0.27 & 0.16 & 0.57 & 0.27 & 0.16 & 0.00 & 3.16 & 2.07 & 3.11\end{array}$ $\begin{array}{llllllllllll}51.37 & 1.43 & 0.56 & 0.41 & 0.23 & 0.56 & 0.41 & 0.23 & 0.00 & 4.03 & 2.68 & 2.96\end{array}$ $\begin{array}{llllllllllll}58.04 & 2.35 & 0.50 & 0.55 & 0.33 & 0.50 & 0.55 & 0.33 & 0.00 & 4.95 & 3.33 & 2.71\end{array}$ $\begin{array}{llllllllllll}65.58 & 3.52 & 0.40 & 0.66 & 0.44 & 0.40 & 0.66 & 0.44 & 0.00 & 5.85 & 3.99 & 2.41\end{array}$ $\begin{array}{llllllllllll}74.09 & 4.90 & 0.29 & 0.72 & 0.54 & 0.29 & 0.72 & 0.54 & 0.00 & 6.64 & 4.60 & 2.07\end{array}$ $\begin{array}{llllllllllll}83.71 & 6.36 & 0.24 & 0.70 & 0.61 & 0.24 & 0.70 & 0.61 & 0.00 & 7.24 & 5.12 & 1.72\end{array}$ $\begin{array}{llllllllllll}94.57 & 7.75 & 0.35 & 0.58 & 0.62 & 0.35 & 0.58 & 0.62 & 0.00 & 7.57 & 5.49 & 1.41\end{array}$ $\begin{array}{llllllllllll}106.85 & 8.90 & 0.70 & 0.37 & 0.55 & 0.70 & 0.37 & 0.55 & 0.00 & 7.59 & 5.69 & 1.13\end{array}$ $\begin{array}{llllllllllll}120.72 & 9.66 & 1.37 & 0.11 & 0.41 & 1.37 & 0.11 & 0.41 & 0.00 & 7.29 & 5.73 & 0.91\end{array}$ $\begin{array}{llllllllllll}136.40 & 9.92 & 2.40 & 0.00 & 0.21 & 2.40 & 0.00 & 0.21 & 0.00 & 6.71 & 5.63 & 0.73\end{array}$ $\begin{array}{llllllllllll}154.10 & 9.63 & 3.76 & 0.00 & 0.03 & 3.76 & 0.00 & 0.03 & 0.00 & 5.94 & 5.45 & 0.58\end{array}$ $\begin{array}{llllllllllll}174.11 & 8.82 & 5.38 & 0.00 & 0.00 & 5.38 & 0.00 & 0.00 & 0.00 & 5.07 & 5.23 & 0.43\end{array}$ $\begin{array}{llllllllllll}196.71 & 7.57 & 7.09 & 0.13 & 0.00 & 7.09 & 0.13 & 0.00 & 0.00 & 4.17 & 5.00 & 0.26\end{array}$ $\begin{array}{llllllllllll}222.25 & 6.03 & 8.67 & 0.66 & 0.01 & 8.67 & 0.66 & 0.01 & 0.00 & 3.33 & 4.77 & 0.05\end{array}$ $\begin{array}{llllllllllll}251.10 & 4.33 & 9.88 & 2.20 & 0.25 & 9.88 & 2.20 & 0.25 & 0.00 & 2.58 & 4.53 & 0.00\end{array}$ 
$\begin{array}{llllllllllll}283.70 & 2.73 & 10.54 & 3.98 & 0.96 & 10.54 & 3.98 & 0.96 & 0.00 & 1.94 & 4.25 & 0.00\end{array}$

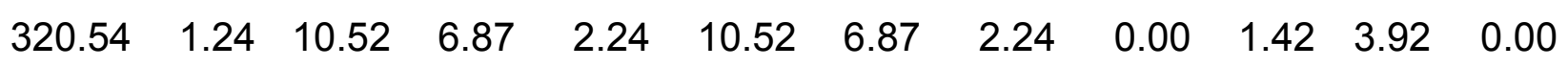
$\begin{array}{llllllllllll}362.15 & 0.04 & 9.81 & 9.72 & 4.06 & 9.81 & 9.72 & 4.06 & 0.00 & 1.01 & 3.53 & 0.00\end{array}$ $\begin{array}{llllllllllll}409.16 & 0.00 & 8.52 & 11.97 & 6.26 & 8.52 & 11.97 & 6.26 & 0.00 & 0.71 & 3.08 & 0.00\end{array}$ $\begin{array}{llllllllllll}462.28 & 0.00 & 6.83 & 13.27 & 8.50 & 6.83 & 13.27 & 8.50 & 0.00 & 0.49 & 2.60 & 0.00\end{array}$ $\begin{array}{llllllllllll}522.30 & 0.00 & 4.94 & 13.07 & 10.38 & 4.94 & 13.07 & 10.38 & 0.00 & 0.34 & 2.09 & 0.00\end{array}$ $\begin{array}{llllllllllll}590.10 & 0.00 & 3.14 & 11.55 & 11.51 & 3.14 & 11.55 & 11.51 & 0.00 & 0.23 & 1.60 & 0.00\end{array}$ $\begin{array}{llllllllllll}666.71 & 0.00 & 1.64 & 9.21 & 11.66 & 1.64 & 9.21 & 11.66 & 0.00 & 0.16 & 1.14 & 0.11\end{array}$ $\begin{array}{llllllllllll}753.26 & 0.00 & 0.22 & 6.04 & 10.83 & 0.22 & 6.04 & 10.83 & 0.25 & 0.12 & 0.74 & 0.51\end{array}$ $\begin{array}{llllllllllll}851.06 & 0.00 & 0.00 & 3.95 & 9.23 & 0.00 & 3.95 & 9.23 & 1.32 & 0.10 & 0.44 & 2.15\end{array}$ $\begin{array}{llllllllllll}961.54 & 0.00 & 0.00 & 1.77 & 7.19 & 0.00 & 1.77 & 7.19 & 5.31 & 0.09 & 0.14 & 4.07\end{array}$ $\begin{array}{llllllllllll}1086.37 & 0.00 & 0.00 & 0.62 & 5.09 & 0.00 & 0.62 & 5.09 & 11.29 & 0.11 & 0.00 & 7.69\end{array}$

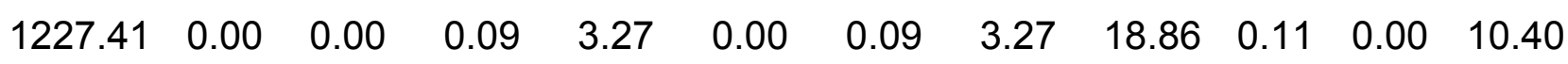
$\begin{array}{llllllllllll}1386.75 & 0.00 & 0.00 & 0.01 & 1.89 & 0.00 & 0.01 & 1.89 & 24.00 & 0.11 & 0.00 & 10.98\end{array}$ $\begin{array}{llllllllllll}1566.78 & 0.00 & 0.00 & 0.00 & 0.97 & 0.00 & 0.00 & 0.97 & 22.38 & 0.10 & 0.00 & 9.27\end{array}$

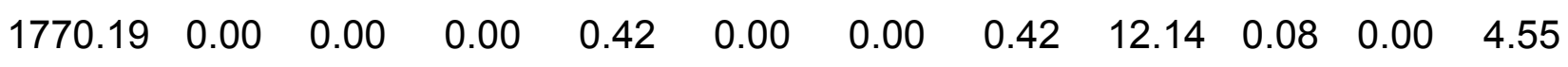
$\begin{array}{llllllllllll}2000.00 & 0.00 & 0.00 & 0.00 & 0.15 & 0.00 & 0.00 & 0.15 & 4.46 & 0.05 & 0.00 & 1.55\end{array}$ 
Table S3. List of ibuprofen CSDs after dissolution.

Particle

Volume

Size

Run Run Run Run Run Run Run Run Run Run Run

\begin{tabular}{cccccccccccc}
$(\mu \mathrm{m})$ & 1 & 2 & 3 & 4 & 5 & 6 & 7 & 8 & 9 & 10 & 11 \\
\hline 0.01 & 0.00 & 0.00 & 0.00 & 0.00 & 0.00 & 0.00 & 0.00 & 0.00 & 0.00 & 0.00 & 0.00
\end{tabular}

$\begin{array}{llllllllllll}0.01 & 0.00 & 0.00 & 0.00 & 0.00 & 0.00 & 0.00 & 0.00 & 0.00 & 0.00 & 0.00 & 0.00\end{array}$

$\begin{array}{llllllllllll}0.01 & 0.00 & 0.00 & 0.00 & 0.00 & 0.00 & 0.00 & 0.00 & 0.00 & 0.00 & 0.00 & 0.00\end{array}$

$\begin{array}{llllllllllll}0.02 & 0.00 & 0.00 & 0.00 & 0.00 & 0.00 & 0.00 & 0.00 & 0.00 & 0.00 & 0.00 & 0.00\end{array}$

$\begin{array}{llllllllllll}0.02 & 0.00 & 0.00 & 0.00 & 0.00 & 0.00 & 0.00 & 0.00 & 0.00 & 0.00 & 0.00 & 0.00\end{array}$

$\begin{array}{llllllllllll}0.02 & 0.00 & 0.00 & 0.00 & 0.00 & 0.00 & 0.00 & 0.00 & 0.00 & 0.00 & 0.00 & 0.00\end{array}$

$\begin{array}{llllllllllll}0.02 & 0.00 & 0.00 & 0.00 & 0.00 & 0.00 & 0.00 & 0.00 & 0.00 & 0.00 & 0.00 & 0.00\end{array}$

$\begin{array}{llllllllllll}0.03 & 0.00 & 0.00 & 0.00 & 0.00 & 0.00 & 0.00 & 0.00 & 0.00 & 0.00 & 0.00 & 0.00\end{array}$

$\begin{array}{llllllllllll}0.03 & 0.00 & 0.00 & 0.00 & 0.00 & 0.00 & 0.00 & 0.00 & 0.00 & 0.00 & 0.00 & 0.00\end{array}$

$\begin{array}{llllllllllll}0.03 & 0.00 & 0.00 & 0.00 & 0.00 & 0.00 & 0.00 & 0.00 & 0.00 & 0.00 & 0.00 & 0.00\end{array}$

$\begin{array}{llllllllllll}0.04 & 0.00 & 0.00 & 0.00 & 0.00 & 0.00 & 0.00 & 0.00 & 0.00 & 0.00 & 0.00 & 0.00\end{array}$

$\begin{array}{llllllllllll}0.04 & 0.00 & 0.00 & 0.00 & 0.00 & 0.00 & 0.00 & 0.00 & 0.00 & 0.00 & 0.00 & 0.00\end{array}$

$\begin{array}{llllllllllll}0.05 & 0.00 & 0.00 & 0.00 & 0.00 & 0.00 & 0.00 & 0.00 & 0.00 & 0.00 & 0.00 & 0.00\end{array}$

$\begin{array}{llllllllllll}0.06 & 0.00 & 0.00 & 0.00 & 0.00 & 0.00 & 0.00 & 0.00 & 0.00 & 0.00 & 0.00 & 0.00\end{array}$

$\begin{array}{llllllllllll}0.06 & 0.00 & 0.00 & 0.00 & 0.00 & 0.00 & 0.00 & 0.00 & 0.00 & 0.00 & 0.00 & 0.00\end{array}$

$\begin{array}{llllllllllll}0.07 & 0.00 & 0.00 & 0.00 & 0.00 & 0.00 & 0.00 & 0.00 & 0.00 & 0.00 & 0.00 & 0.00\end{array}$

$\begin{array}{llllllllllll}0.08 & 0.00 & 0.00 & 0.00 & 0.00 & 0.00 & 0.00 & 0.00 & 0.00 & 0.00 & 0.00 & 0.00\end{array}$

$\begin{array}{llllllllllll}0.09 & 0.00 & 0.00 & 0.00 & 0.00 & 0.00 & 0.00 & 0.00 & 0.00 & 0.00 & 0.00 & 0.00\end{array}$

$\begin{array}{llllllllllll}0.10 & 0.00 & 0.00 & 0.00 & 0.00 & 0.00 & 0.00 & 0.00 & 0.00 & 0.00 & 0.00 & 0.00\end{array}$

$\begin{array}{llllllllllll}0.11 & 0.00 & 0.00 & 0.00 & 0.00 & 0.00 & 0.00 & 0.00 & 0.00 & 0.00 & 0.00 & 0.00\end{array}$

$\begin{array}{llllllllllll}0.13 & 0.00 & 0.00 & 0.00 & 0.00 & 0.00 & 0.00 & 0.00 & 0.00 & 0.00 & 0.00 & 0.00\end{array}$

$\begin{array}{llllllllllll}0.15 & 0.00 & 0.00 & 0.00 & 0.00 & 0.00 & 0.00 & 0.00 & 0.00 & 0.00 & 0.00 & 0.00\end{array}$

$\begin{array}{llllllllllll}0.17 & 0.00 & 0.00 & 0.00 & 0.00 & 0.00 & 0.00 & 0.00 & 0.00 & 0.00 & 0.00 & 0.00\end{array}$

$\begin{array}{llllllllllll}0.19 & 0.00 & 0.00 & 0.00 & 0.00 & 0.00 & 0.00 & 0.00 & 0.00 & 0.00 & 0.00 & 0.00\end{array}$ 
$\begin{array}{llllllllllll}0.21 & 0.00 & 0.00 & 0.00 & 0.00 & 0.00 & 0.00 & 0.00 & 0.00 & 0.00 & 0.00 & 0.00\end{array}$

$\begin{array}{llllllllllll}0.24 & 0.00 & 0.00 & 0.00 & 0.00 & 0.00 & 0.00 & 0.00 & 0.00 & 0.00 & 0.00 & 0.00\end{array}$

$\begin{array}{llllllllllll}0.27 & 0.00 & 0.00 & 0.00 & 0.00 & 0.00 & 0.00 & 0.00 & 0.00 & 0.00 & 0.00 & 0.00\end{array}$

$\begin{array}{llllllllllll}0.30 & 0.00 & 0.00 & 0.00 & 0.00 & 0.00 & 0.00 & 0.00 & 0.00 & 0.00 & 0.00 & 0.00\end{array}$

$\begin{array}{llllllllllll}0.34 & 0.00 & 0.00 & 0.00 & 0.00 & 0.00 & 0.00 & 0.00 & 0.00 & 0.00 & 0.00 & 0.00\end{array}$

$\begin{array}{llllllllllll}0.39 & 0.00 & 0.00 & 0.00 & 0.00 & 0.00 & 0.00 & 0.00 & 0.00 & 0.00 & 0.00 & 0.00\end{array}$

$\begin{array}{llllllllllll}0.44 & 0.00 & 0.00 & 0.00 & 0.00 & 0.00 & 0.00 & 0.00 & 0.00 & 0.00 & 0.00 & 0.00\end{array}$

$\begin{array}{llllllllllll}0.50 & 0.00 & 0.00 & 0.00 & 0.00 & 0.00 & 0.00 & 0.00 & 0.00 & 0.00 & 0.00 & 0.00\end{array}$

$\begin{array}{llllllllllll}0.56 & 0.00 & 0.00 & 0.00 & 0.00 & 0.00 & 0.00 & 0.00 & 0.00 & 0.00 & 0.00 & 0.00\end{array}$

$\begin{array}{llllllllllll}0.63 & 0.00 & 0.00 & 0.00 & 0.00 & 0.00 & 0.00 & 0.00 & 0.00 & 0.00 & 0.00 & 0.00\end{array}$

$\begin{array}{llllllllllll}0.72 & 0.00 & 0.00 & 0.00 & 0.00 & 0.00 & 0.00 & 0.00 & 0.00 & 0.00 & 0.00 & 0.00\end{array}$

$\begin{array}{llllllllllll}0.81 & 0.00 & 0.00 & 0.00 & 0.00 & 0.00 & 0.00 & 0.00 & 0.00 & 0.00 & 0.00 & 0.00\end{array}$

$\begin{array}{llllllllllll}0.91 & 0.00 & 0.00 & 0.00 & 0.00 & 0.00 & 0.00 & 0.00 & 0.00 & 0.00 & 0.00 & 0.00\end{array}$

$\begin{array}{llllllllllll}1.03 & 0.00 & 0.00 & 0.00 & 0.00 & 0.00 & 0.00 & 0.00 & 0.00 & 0.00 & 0.00 & 0.00\end{array}$

$\begin{array}{llllllllllll}1.17 & 0.00 & 0.00 & 0.00 & 0.00 & 0.00 & 0.00 & 0.00 & 0.00 & 0.00 & 0.00 & 0.00\end{array}$

$\begin{array}{llllllllllll}1.32 & 0.00 & 0.00 & 0.00 & 0.00 & 0.00 & 0.00 & 0.00 & 0.00 & 0.01 & 0.00 & 0.00\end{array}$

$\begin{array}{llllllllllll}1.49 & 0.00 & 0.00 & 0.00 & 0.00 & 0.00 & 0.00 & 0.00 & 0.00 & 0.04 & 0.00 & 0.00\end{array}$

$\begin{array}{llllllllllll}1.68 & 0.00 & 0.00 & 0.00 & 0.00 & 0.00 & 0.00 & 0.00 & 0.00 & 0.07 & 0.00 & 0.00\end{array}$

$\begin{array}{llllllllllll}1.90 & 0.00 & 0.00 & 0.00 & 0.00 & 0.00 & 0.00 & 0.00 & 0.00 & 0.08 & 0.00 & 0.00\end{array}$

$\begin{array}{llllllllllll}2.15 & 0.00 & 0.00 & 0.00 & 0.00 & 0.00 & 0.00 & 0.00 & 0.00 & 0.10 & 0.05 & 0.00\end{array}$

$\begin{array}{llllllllllll}2.43 & 0.00 & 0.00 & 0.00 & 0.00 & 0.00 & 0.00 & 0.00 & 0.00 & 0.11 & 0.06 & 0.01\end{array}$

$\begin{array}{llllllllllll}2.74 & 0.00 & 0.00 & 0.00 & 0.00 & 0.00 & 0.00 & 0.00 & 0.00 & 0.12 & 0.06 & 0.02\end{array}$

$\begin{array}{llllllllllll}3.10 & 0.00 & 0.00 & 0.00 & 0.00 & 0.00 & 0.00 & 0.00 & 0.00 & 0.12 & 0.06 & 0.02\end{array}$

$\begin{array}{llllllllllll}3.50 & 0.00 & 0.00 & 0.00 & 0.00 & 0.00 & 0.00 & 0.00 & 0.00 & 0.12 & 0.06 & 0.02\end{array}$

$\begin{array}{llllllllllll}3.96 & 0.00 & 0.00 & 0.00 & 0.00 & 0.00 & 0.00 & 0.00 & 0.00 & 0.11 & 0.06 & 0.02\end{array}$

$\begin{array}{llllllllllll}4.47 & 0.00 & 0.00 & 0.00 & 0.00 & 0.00 & 0.00 & 0.00 & 0.00 & 0.11 & 0.06 & 0.00\end{array}$

$\begin{array}{llllllllllll}5.05 & 0.00 & 0.00 & 0.00 & 0.00 & 0.00 & 0.00 & 0.00 & 0.00 & 0.11 & 0.06 & 0.00\end{array}$

$\begin{array}{llllllllllll}5.71 & 0.00 & 0.00 & 0.00 & 0.00 & 0.00 & 0.00 & 0.00 & 0.00 & 0.12 & 0.05 & 0.00\end{array}$

$\begin{array}{llllllllllll}6.45 & 0.00 & 0.00 & 0.00 & 0.00 & 0.00 & 0.00 & 0.00 & 0.00 & 0.14 & 0.06 & 0.00\end{array}$ $\begin{array}{llllllllllll}7.29 & 0.00 & 0.00 & 0.00 & 0.00 & 0.00 & 0.00 & 0.00 & 0.00 & 0.15 & 0.06 & 0.00\end{array}$ 
$\begin{array}{llllllllllll}8.23 & 0.00 & 0.00 & 0.00 & 0.00 & 0.00 & 0.00 & 0.00 & 0.00 & 0.18 & 0.07 & 0.00\end{array}$ $\begin{array}{llllllllllll}9.30 & 0.04 & 0.00 & 0.00 & 0.00 & 0.00 & 0.00 & 0.00 & 0.00 & 0.20 & 0.07 & 0.00\end{array}$ $\begin{array}{llllllllllll}10.51 & 0.09 & 0.00 & 0.00 & 0.00 & 0.00 & 0.00 & 0.00 & 0.00 & 0.21 & 0.08 & 0.01\end{array}$ $\begin{array}{llllllllllll}11.87 & 0.14 & 0.00 & 0.00 & 0.00 & 0.00 & 0.00 & 0.00 & 0.00 & 0.23 & 0.09 & 0.04\end{array}$ $\begin{array}{llllllllllll}13.42 & 0.18 & 0.00 & 0.00 & 0.00 & 0.00 & 0.00 & 0.00 & 0.00 & 0.24 & 0.11 & 0.06\end{array}$ $\begin{array}{llllllllllll}15.16 & 0.22 & 0.00 & 0.00 & 0.00 & 0.00 & 0.00 & 0.00 & 0.00 & 0.26 & 0.12 & 0.07\end{array}$ $\begin{array}{llllllllllll}17.12 & 0.25 & 0.00 & 0.00 & 0.00 & 0.00 & 0.00 & 0.00 & 0.00 & 0.29 & 0.15 & 0.09\end{array}$ $\begin{array}{llllllllllll}19.35 & 0.25 & 0.02 & 0.00 & 0.00 & 0.03 & 0.00 & 0.00 & 0.00 & 0.36 & 0.18 & 0.11\end{array}$ $\begin{array}{llllllllllll}21.86 & 0.23 & 0.08 & 0.00 & 0.00 & 0.11 & 0.00 & 0.00 & 0.00 & 0.49 & 0.23 & 0.15\end{array}$ $\begin{array}{llllllllllll}24.70 & 0.19 & 0.14 & 0.00 & 0.00 & 0.17 & 0.00 & 0.00 & 0.00 & 0.70 & 0.31 & 0.20\end{array}$ $\begin{array}{llllllllllll}27.90 & 0.16 & 0.20 & 0.00 & 0.00 & 0.23 & 0.00 & 0.00 & 0.00 & 1.02 & 0.42 & 0.28\end{array}$ $\begin{array}{llllllllllll}31.53 & 0.17 & 0.25 & 0.01 & 0.00 & 0.26 & 0.04 & 0.00 & 0.00 & 1.47 & 0.58 & 0.38\end{array}$ $\begin{array}{llllllllllll}35.62 & 0.26 & 0.28 & 0.07 & 0.00 & 0.28 & 0.09 & 0.00 & 0.00 & 2.07 & 0.78 & 0.51\end{array}$ $\begin{array}{llllllllllll}40.24 & 0.48 & 0.28 & 0.14 & 0.00 & 0.25 & 0.14 & 0.01 & 0.00 & 2.81 & 1.05 & 0.68\end{array}$ $\begin{array}{llllllllllll}45.47 & 0.88 & 0.23 & 0.21 & 0.06 & 0.18 & 0.19 & 0.06 & 0.00 & 3.68 & 1.37 & 0.88\end{array}$ $\begin{array}{llllllllllll}51.37 & 1.51 & 0.16 & 0.25 & 0.09 & 0.10 & 0.23 & 0.10 & 0.00 & 4.64 & 1.75 & 1.12\end{array}$ $\begin{array}{llllllllllll}58.04 & 2.39 & 0.09 & 0.27 & 0.12 & 0.01 & 0.25 & 0.14 & 0.00 & 5.61 & 2.18 & 1.40\end{array}$ $\begin{array}{llllllllllll}65.58 & 3.48 & 0.06 & 0.25 & 0.16 & 0.01 & 0.23 & 0.19 & 0.00 & 6.51 & 2.62 & 1.70\end{array}$ $\begin{array}{llllllllllll}74.09 & 4.74 & 0.14 & 0.18 & 0.18 & 0.11 & 0.18 & 0.21 & 0.05 & 7.26 & 3.07 & 2.02\end{array}$ $\begin{array}{llllllllllll}83.71 & 6.06 & 0.41 & 0.08 & 0.18 & 0.39 & 0.09 & 0.21 & 0.08 & 7.77 & 3.49 & 2.35\end{array}$ $\begin{array}{llllllllllll}94.57 & 7.33 & 0.93 & 0.00 & 0.15 & 0.97 & 0.00 & 0.17 & 0.11 & 7.98 & 3.87 & 2.69\end{array}$ $\begin{array}{llllllllllll}106.85 & 8.39 & 1.77 & 0.00 & 0.09 & 1.94 & 0.00 & 0.10 & 0.14 & 7.84 & 4.20 & 3.04\end{array}$ $\begin{array}{llllllllllll}120.72 & 9.11 & 2.92 & 0.00 & 0.02 & 3.33 & 0.00 & 0.02 & 0.16 & 7.37 & 4.47 & 3.40\end{array}$ $\begin{array}{llllllllllll}136.40 & 9.40 & 4.35 & 0.00 & 0.00 & 5.06 & 0.00 & 0.00 & 0.16 & 6.63 & 4.70 & 3.78\end{array}$ $\begin{array}{llllllllllll}154.10 & 9.22 & 5.94 & 0.01 & 0.00 & 6.97 & 0.09 & 0.00 & 0.14 & 5.70 & 4.91 & 4.20\end{array}$ $\begin{array}{llllllllllll}174.11 & 8.57 & 7.55 & 0.36 & 0.00 & 8.82 & 0.72 & 0.00 & 0.10 & 4.70 & 5.09 & 4.63\end{array}$ $\begin{array}{llllllllllll}196.71 & 7.55 & 8.98 & 1.23 & 0.00 & 10.31 & 1.75 & 0.00 & 0.04 & 3.71 & 5.26 & 5.05\end{array}$ $\begin{array}{llllllllllll}222.25 & 6.26 & 10.02 & 2.75 & 0.32 & 11.19 & 3.24 & 0.12 & 0.00 & 2.79 & 5.39 & 5.44\end{array}$ $\begin{array}{llllllllllll}251.10 & 4.86 & 10.52 & 4.86 & 1.01 & 11.26 & 5.11 & 0.69 & 0.00 & 2.00 & 5.45 & 5.72\end{array}$ $\begin{array}{llllllllllll}283.70 & 3.49 & 10.39 & 7.35 & 1.99 & 10.52 & 7.15 & 1.67 & 0.00 & 1.36 & 5.41 & 5.87\end{array}$ 


$\begin{array}{cccccccccccc}320.54 & 2.26 & 9.62 & 9.82 & 3.27 & 9.09 & 9.10 & 2.99 & 0.07 & 0.86 & 5.27 & 5.86 \\ 362.15 & 1.26 & 8.31 & 11.79 & 4.74 & 7.22 & 10.65 & 4.59 & 0.33 & 0.51 & 5.00 & 5.68 \\ 409.16 & 0.52 & 6.66 & 12.81 & 6.26 & 5.21 & 11.52 & 6.31 & 0.90 & 0.28 & 4.62 & 5.34 \\ 462.28 & 0.07 & 4.82 & 12.66 & 7.67 & 3.34 & 11.54 & 7.94 & 1.81 & 0.14 & 4.14 & 4.88 \\ 522.30 & 0.00 & 3.10 & 11.35 & 8.78 & 1.83 & 10.72 & 9.25 & 3.09 & 0.07 & 3.58 & 4.33 \\ 590.10 & 0.00 & 1.54 & 9.21 & 9.46 & 0.75 & 9.19 & 10.05 & 4.69 & 0.02 & 2.98 & 3.74 \\ 666.71 & 0.00 & 0.22 & 6.70 & 9.65 & 0.09 & 7.23 & 10.26 & 6.50 & 0.03 & 2.35 & 3.15 \\ 753.26 & 0.00 & 0.02 & 4.28 & 9.34 & 0.00 & 5.15 & 9.87 & 8.34 & 0.04 & 1.74 & 2.61 \\ 851.06 & 0.00 & 0.00 & 2.30 & 8.60 & 0.00 & 3.21 & 8.95 & 10.00 & 0.05 & 1.17 & 2.12 \\ 961.54 & 0.00 & 0.00 & 0.91 & 7.54 & 0.00 & 1.65 & 7.66 & 11.22 & 0.06 & 0.68 & 1.71 \\ 1086.37 & 0.00 & 0.00 & 0.15 & 6.29 & 0.00 & 0.52 & 6.17 & 11.80 & 0.07 & 0.27 & 1.37 \\ 1227.41 & 0.00 & 0.00 & 0.00 & 5.01 & 0.00 & 0.00 & 4.70 & 11.58 & 0.08 & 0.03 & 1.10 \\ 1386.75 & 0.00 & 0.00 & 0.00 & 3.78 & 0.00 & 0.00 & 3.35 & 10.49 & 0.08 & 0.00 & 0.86 \\ 1566.78 & 0.00 & 0.00 & 0.00 & 2.69 & 0.00 & 0.00 & 2.25 & 8.73 & 0.07 & 0.00 & 0.64 \\ 1770.19 & 0.00 & 0.00 & 0.00 & 1.67 & 0.00 & 0.00 & 1.32 & 6.05 & 0.05 & 0.00 & 0.42 \\ 2000.00 & 0.00 & 0.00 & 0.00 & 0.87 & 0.00 & 0.00 & 0.65 & 3.39 & 0.03 & 0.00 & 0.23\end{array}$

Table S4. Particle size of ibuprofen pre and post-dissolution process.

\begin{tabular}{lccccc}
\hline Measure Stage & $\begin{array}{c}D[3,2]^{*}, \\
\mu \mathrm{m}\end{array}$ & $\begin{array}{c}D[4,3]^{* *} \\
\mu \mathrm{m}\end{array}$ & $D 10, \mu \mathrm{m}$ & $D 50, \mu \mathrm{m}$ & $D 90, \mu \mathrm{m}$ \\
\hline Pre-dissolution & 157.273 & 256.622 & 83.173 & 210.773 & 502.081 \\
& 168.274 & 269.748 & 88.074 & 224.355 & 521.629 \\
Post-dissolution & & & & & \\
\hline
\end{tabular}

